\title{
Quantum efficiency of self-assembled quantum dots determined by a modified optical local density of states
}

\author{
Johansen, Jeppe; Stobbe, Søren; Nikolaev, I.S.; Lund-Hansen, Toke; Kristensen, Philip Trøst; Hvam, \\ Jørn Märcher; Vos, Willem L.; Lodahl, Peter
}

\section{Published in:}

Proceedings CLEO/QELS

Link to article, DOI:

10.1109/QELS.2007.4431638

Publication date:

2007

Document Version

Publisher's PDF, also known as Version of record

Link back to DTU Orbit

Citation (APA):

Johansen, J., Stobbe, S., Nikolaev, I. S., Lund-Hansen, T., Kristensen, P. T., Hvam, J. M., Vos, W. L., \& Lodahl, P. (2007). Quantum efficiency of self-assembled quantum dots determined by a modified optical local density of states. In Proceedings CLEO/QELS (pp. 1-2). IEEE. https://doi.org/10.1109/QELS.2007.4431638

\section{General rights}

Copyright and moral rights for the publications made accessible in the public portal are retained by the authors and/or other copyright owners and it is a condition of accessing publications that users recognise and abide by the legal requirements associated with these rights.

- Users may download and print one copy of any publication from the public portal for the purpose of private study or research.

- You may not further distribute the material or use it for any profit-making activity or commercial gain

- You may freely distribute the URL identifying the publication in the public portal 


\title{
Quantum Efficiency of Self-Assembled Quantum Dots Determined by a Modified Optical Local Density of States
}

\author{
Jeppe Johansen ${ }^{1}$, Søren Stobbe ${ }^{1}$, Ivan S. Nikolaev ${ }^{2,3}$, Toke Lund-Hansen ${ }^{1}$, Philip T. Kristensen ${ }^{1}$, \\ Jørn M. Hvam ${ }^{1}$, Willem L. Vos ${ }^{2,3}$, Peter Lodahl ${ }^{1}$ \\ ${ }^{1}$ COM॰DTU, Department of Communications, Optics, and Materials \\ Nano $\bullet T U$, Technical University of Denmark, DTU - Building 345V, DK-2800 Kgs. Lyngby, Denmark \\ Phone:+4545253731,FAX: +4545936581,E-mail: jjo@com.dtu.dk \\ ${ }^{2}$ FOM Institute for Atomic and Molecular Physics (AMOLF), 1098 SJ Amsterdam, The Netherlands \\ ${ }^{3}$ Complex Photonic Systems, MESA ${ }^{+}$Research Institute, University of Twente, 7500 AE Enschede, The Netherlands
}

\begin{abstract}
We have measured time-resolved spontaneous emission from quantum dots near a dielectric interface with known photonic local density of states. We thus experimentally determine the quantum efficiency and the dipole moment, important for quantum optics.

(C)2006 Optical Society of America

OCIS codes: (270.0270), (250.5230), (270.5580).
\end{abstract}

\section{Introduction}

Control of spontaneous emission from an excited emitter is central in quantum optics. Recent advancements in solid-state quantum optics include the demonstration of strong coupling between a single quantum dot (QD) and the optical mode of a cavity [1,2], and the control of spontaneous emission decay rates using photonic crystals [3]. The rate of spontaneous emission can be controlled through the number of optical modes available for emission, which is quantified by the local density of states (LDOS). Moreover, as the non-radiative decay rate is unaffected by a modification of the LDOS, quantum optics experiments require high internal quantum efficiency of the emitter.

We present quantitative measurements of the radiative and non-radiative decay rates, the quantum efficiency, and the dipole moment of self-assembled InAs/GaAs QDs. As the LDOS can be calculated exactly, these quantities can be determined from time-resolved measurements of spontaneous emission at different distances to an interface. Time-resolved measurements will be essential for an unambiguous proof of strong coupling of a single QD to a single photon. So far the only signs of strong coupling have been $\mathrm{CW}$-measurements where a clear Rabi-splitting has been observed [1,2].

\section{Discussion}

We have performed time- and spectrally-resolved measurements of spontaneous emission from ensembles of selfassembled InAs/GaAs QDs near an air-GaAs interface. Due to reflections of the electromagnetic field from the airGaAs interface, the LDOS oscillates as the distance to the interface is varied. This oscillation of the LDOS is observed in the measured decay rates (Fig. 1(a), solid dots). The data shown in Figure 1 are taken at the energy corresponding to the ground state transition of the QDs.

Both radiative as well as non-radiative processes can lead to a decay of the excited QDs. The non-radiative decay rate is unaffected by modifications of the LDOS, while the radiative decay rate is directly proportional to the normalized LDOS. Thus the measured decay rate can be expressed as

$$
\Gamma_{\text {tot }}(\omega, r)=\Gamma_{\text {rad }-\mathrm{hom}}(\omega) \frac{\rho(\omega, r)}{\rho_{\text {hom }}(\omega)}+\Gamma_{\text {non-rad }}(\omega),
$$

where $\Gamma_{\text {rad-hom }}(\omega)$ is the radiative decay rate in a homogeneous medium, $\rho(\omega, \mathrm{r}) / \rho_{\text {hom }}(\omega)$ the position dependent LDOS near the interface - normalized to the LDOS in a homogenous medium, and $\Gamma_{\text {non-rad }}(\omega)$ is the non-radiative decay rate. In order to quantify the radiative and non-radiative decay rates the measured decay rates are plotted as a function of the calculated normalized LDOS (Fig. 1(b)). The slope of the linear fit gives the radiative decay rate, while the intersection with the ordinate gives the non-radiative decay rate. We find a radiative decay rate $\Gamma_{\text {rad-hom }}$ of $0.85 \pm 0.04 \mathrm{~ns}^{-1}$ and non-radiative decay rate $\Gamma_{\text {non-rad }}$ of $0.18 \pm 0.04 \mathrm{~ns}^{-1}$. This corresponds to a high quantum efficiency 


\section{QMK5.pdf}

of $82 \%$. The radiative decay rate for a quantum dot in a homogenous medium can be calculated from Fermi's Golden Rule [4],

$$
\Gamma_{r a d-\text { hom }}=\frac{d^{2} n \omega_{b u l k}^{2} \omega}{3 \hbar \varepsilon_{0} \pi c^{3}}
$$

Here $d$ is the dipole moment, $n$ the refractive index, $\omega_{\text {bulk }}$ the frequency of the band gap for the bulk semiconductor, and $\omega$ the frequency of the QD transition. From this we find dipole moment of $d \frac{\omega_{b u l k}}{\omega}=9.7 \pm 0.2 \times 10^{-29} \mathrm{~m} \mathrm{C}$, corresponding to an oscillator strength of $11.7 \pm 0.5$.
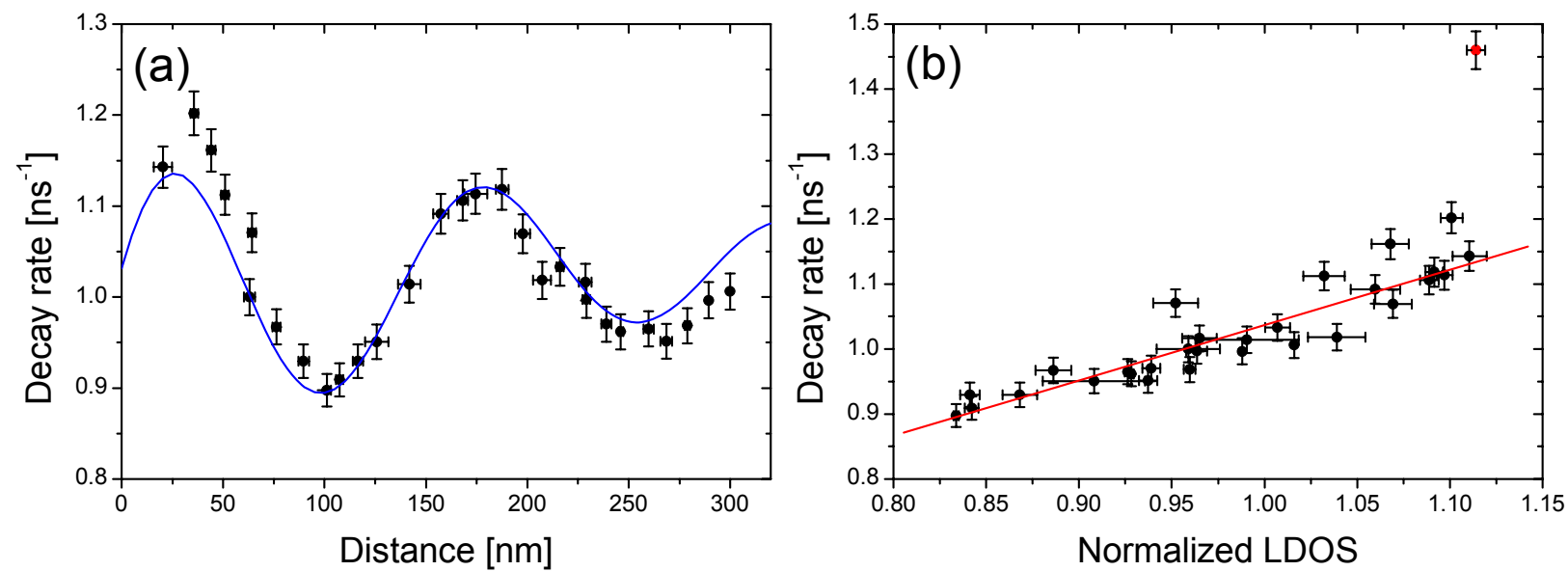

Figure 1 (a) Measured decay rates of spontaneous emission from QDs for different distances to the air-GaAs interface (solid dots). The solid line is the theoretical decay rate calculated using the deduced radiative and non-radiative decay rates. (b) The measured decay rates plotted against the calculated LDOS. The solid line is a linear fit to the data points. The single data point (open dot) in the upper right corner, which was taken very close to the interface, is disregarded since surface recombinations might have led to the increase in decay rate.

From the extracted values, the decay rates can be calculated as a function of the distance (Fig. 1(a), solid line). The observed modification of the decay rates is very well described by theory assuming that the dipoles have a fixed orientation parallel to the interface. This is in agreement with results in literature obtained from absorption measurements where the ground state is found to be primarily TE polarized [5].

The quantum efficiencies and dipole moments of excited states of the QDs are extracted in a similar manner from measurements performed at higher transition energies. As these transitions often have both TE and TM polarized components, the observed decays have a more complex dynamical behavior, since these components couples to different projections of the LDOS.

Our results are important for a quantitative understanding of the decay of QDs in complex nanophotonic structures such as photonic crystals and nanocavities.

\section{References}

[1] T. Yoshie, A. Scherer, J. Hendrickson, G. Khitrova, H. M. Gibbs, G. Rupper, C. Ell, O. B. Shchekin, and D. G. Deppe, "Vacuum Rabi splitting with a single quantum dot in a photonic crystal nanocavity”, Nature, 432, 200 (2004).

[2] J.P. Reithmaier, G. Sek, A. Löffler, C. Hofmann, S. Kuhn, S. Reitzenstein, L.V. Keldysh, V.D. Kulakovskii, T.L. Reinecke, and A. Forchel, "Strong coupling in a single quantum dot - semiconductor microcavity system", Nature 432, 197 (2004)

[3] P. Lodahl, A.F. van Driel, I.S. Nikolaev, A. Irman, K. Overgaag, D. Vanmaekelbergh, and W.L. Vos, "Controlling the dynamics of spontaneous emission from quantum dots by photonic crystals", Nature 430, 654 (2004).

[4] A.F. van Driel, G. Allan, C. Delerue, P. Lodahl, W.L. Vos, and D. Vanmaekelbergh, "Frequency-dependent spontaneous emission from CdSe and CdTe nanocrystals: Influence of dark states", Phys Rev Lett 95, 236804 (2005)

[5] S. Cortez, O. Krebs, P. Voisin, and J.M. Gerard, "Polarization of the interband optical dipole in InAs/GaAs self-organized quantum dots", Phys Rev B 63, 233306 (2001). 